\title{
Is magnetar a fact or fiction to us?
}

\author{
H. Tong ${ }^{1}$ and R. X. $\mathrm{Xu}^{2}$ \\ ${ }^{1}$ Xinjiang Astronomical Observatory, Chinese Academy of Sciences, Urumqi, Xinjiang 830011, \\ China; Email: tonghao@xao.ac.cn \\ ${ }^{2}$ School of Physics and State Key Laboratory of Nuclear Physics and Technology, Peking \\ University, Beijing 100871, China; Email: r.x.xu@pku.edu.cn
}

\begin{abstract}
The key point of studying AXPs/SGRs (anomalous X-ray pulsars/soft gamma-ray repeaters) is relevant to the energy budget. Historically, rotation was thought to be the only free energy of pulsar until the discovery of accretion power in X-ray binaries. AXPs/SGRs could be magnetars if they are magnetism-powered, but would alternatively be quark-star/fallback-disk systems if more and more observations would hardly be understood in the magnetar scenario.
\end{abstract}

Keywords. pulsars: general, stars: magnetars, stars: neutron

\section{Introduction}

Anomalous X-ray pulsars/soft gamma-ray repeaters (AXPs/SGRs) are focused because of their huge energy release and peculiar behavior, suggesting that extra energy sources besides spin and accretion powers should play an important role there. Magnetic energy would be one of the candidates, which was initially proposed. However, this viewpoint could be challenged by more and more observations. It is worth noting here that, to solve the energy budget would be a key to understand the nature of compact stars, the equation state of dense matter at supra-nuclear density.

Let's have a brief note on the history. Rapid rotation was generally thought to be the only energy source for pulsar emission soon after the discovery of radio pulsars (Manchester \& Taylor 1977) until the discovery of accretion-powered pulsars in X-ray binaries (Pringle \& Rees 1972). However, AXPs/SGRs have long spin periods (thus low spindown power, their X-ray luminosities are much larger than their spindown powers) and no binary companions, which rules out spin and accretion in binary system as the power sources for the emission. It was then proposed that SGR-like bursts as well as the persistent X-ray emission could plausibly be the result of field decay of ultra-magnetic neutron stars if MHD dynamo action in the proto-stars is very effective in case that the objects spin initially at periods of $\sim 1 \mathrm{~ms}$ (e.g., Duncan \& Thompson 1992). Because of the starquakes in the crusts of normal neutron stars, a self-induction electric field is created. The strong electric field could initiate avalanches of pair creation in the magnetosphere and certainly accelerate particles, resulting in a so-called magnetar corona (Beloborodov \& Thompson 2006), from which high-energy bursts could be observed. The power source of AXPs/SGRs is actually the magnetic energy through field-reconnection there. This magnetar model is very popular nowadays in the astrophysical community.

Unfortunately, many predictions (see following sections) in the magnetar model have never been confirmed with certainty yet by later observations, and we then have to revisit the real nature of AXP/SGRs: are they really magnetars or alternatives? We are repeatedly asking these questions (Xu 2007, Tong \& Xu 2011), and try to conclude that AXP/SGRs might not be magnetars but could be quark-star/fallback-disk systems. 


\section{Evidence for magnetars?}

The periods and period derivatives of different kinds of pulsar-like stars are shown in Fig. 1. We are summarizing possible observational evidence for magnetars below.

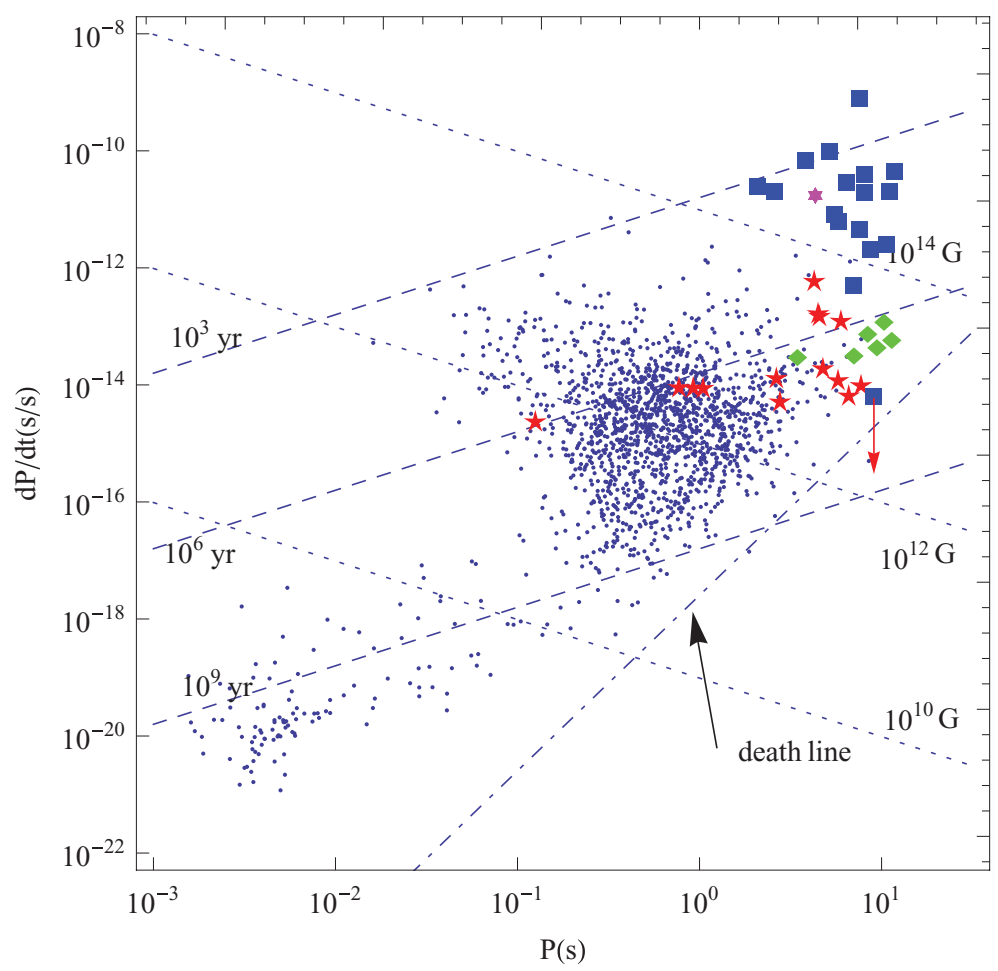

Figure 1. P-P diagram. Squares are for AXPs and SGRs, the six-pointed-star is for the radio loud magnetar (from McGill online), diamonds for X-ray dim isolated neutron stars (XDINSs), stars for rotating radio transients (RRATs), and dots for normal pulsars (from the ATNF).

- Strong dipole magnetic B-fields, measured by assuming magnetic dipole braking.

- Surface B-fields inferred from cyclotron absorptions if protons are responsible.

- Magnetic confinement of the giant flare tails after sudden release of magnetic energy.

- Magnetic suppression of scattering cress-section account for luminosity $L_{x} \sim 10^{7} L_{\mathrm{Edd}}$.

- SGR-like bursts from the high-B PSR J1846-0258 (but, how about the low-B SGRs?).

- Energy of both persistent \& burst emissions, spectral modeling (but modeldependent).

\section{Challenges to the magnetar idea}

- Energetic supernova remnants associated with magnetars due to initial faster spins (initial period $\left.P_{0} \sim 1 \mathrm{~ms}\right)$ and higher B-fields $\left(B_{0} \sim 10^{14-16} \mathrm{G}\right)$.

- A proto-neutron star with small $P_{0}$ and high $B_{0}$ may result in a large kick velocity.

- No radio emissions because of high B-field (but transient emissions are detected).

- Energetic gamma-rays from outer gaps, to be detectable by Fermi-LAT.

- The unexpected discovery of a low-B SGRs, $B<7 \times 10^{12} \mathrm{G}$.

- Unexpected existence of transient magnetars and high-B PSRs.

- Magnetar free precession caused by higher mountains due to higher B-field. 


\section{A solution?}

In view of the failed predictions of the magnetar model, listed in $\S 3$, we are obliged to think about alternative scenarios of AXP/SGRs. We note that the peculiar manifestations of AXP/SGRs would relate closely their inner structures, i.e., the physics of dense matter at supranuclear density. It is well known that baryons of an evolved massive star should be significantly compressed during core-collapse supernova, but the nature of this compressed baryonic matter is still a matter of debate due to strong non-perturbative effects of the fundamental color interaction. At a few nuclear densities, neutron stars containing almost only neutrons (and other hadrons) are presumed to born soon after supernova, while the remnant core might be composed by quark matter if the quark degrees of freedom would not be negligible there. Nonetheless, an emergence of quark-cluster state would be possible if the coupling between quarks inside compact stars is still very strong, forming a solid quark matter star (Xu 2003). An accretion-induced quake model for AXP/SGRs is then proposed (Xu et al. 2006), in which the energy release during star quakes can be estimated as high as $\Delta E=\left(G M^{2} / R\right)(\Delta R / R) \sim 5 \times 10^{47}|\Delta R / R| /\left(10^{-6}\right)$ ergs, to be enough to power the SGR giant flares.

Strong ejection (or wind) would certainly occur during accretion-induced energy release and/or a star quake-induced burst, and the central star may undergo a period of wind braking. If AXP/SGRs are braked by wind instead of magnetic dipole radiation, then their magnetosphere structure is different from that of normal pulsars. This may explain the non-detection in Fermi observations of magnetars (Tong et al. 2012). The extended emission around AXP 1E 1547.0-5408 may be a magnetism-powered pulsar wind nebula. Under the wind braking scenario, a braking index smaller than three is expected.

How can one finally differentiate the magnetar model and the quark-star/fallback-disk model? X-ray polarimetry may play an important role in identifying the real equation of state of dense matter at supranuclear density ( $\mathrm{Lu}$ et al. 2012). We are expecting such an advanced facility to give a final answer to this 80-year-longstanding problem.

\section{Conclusions}

Both the magnetar model and the fallback disk model for AXP/SGRs are discussed. AXP/SGRs could be magnetars, but the origin and presence of strong dipole field are challenged by recent observations. Alternatively, both the bursts and persistent emissions of AXP/SGRs could be understandable in the quark-star/fallback-disk model.

\section{Acknowledgments}

This work is supported by 973-projects (2012CB821800,2009CB82- 4800), NSFC (Grant Nos. 11103021, 10935001, 10973002), the Programme of the Light in China's Western Region and the John Templeton Foundation.

\section{References}

Beloborodov, A. M. \& Thompson, C. 2006, ApJ, 657, 967

Duncan, R. C. \& Thompson, C. 1992, ApJ, 392, L9

Lu, J. G., Feng, H., \& Xu, R. X. 2012, to be submitted

Manchester, R. N. \& Taylor, J. H. Pulsars, (Freeman, San Francisco, 1977)

Pringle, J. E. \& Rees, M. J. 1972, A\&AA, 21, 1

Tong, H. \& Xu, R. X. 2011, Int. Jour. Mod. Phys. E, 20, 15

Tong, H., Xu, R. X., Song, L. M., \& Qiao, G. J. 2012, arXiv:1205.1626

Xu, R. X. 2003, ApJ, 596, L59

Xu, R. X. 2007, Advances in Space Research, 40, 1453

Xu, R. X., Tao, D. J., \& Yang, Y. 2006, MNRAS, 373, L85 